\title{
Use of Ceramic Scaffolds as Inductors of Extramedullar Hematopoiesis
}

\author{
H. Schechtman ${ }^{1, a}$, M. Pelajo-Machado ${ }^{1, b}$, M.H. Prado da Silva ${ }^{2, c}$, \\ C.A. Müller ${ }^{1, d}$, R.G. de Andrade ${ }^{1, e}$ and H.L. Lenzi ${ }^{1, f}$ \\ ${ }^{1}$ Fundação Oswaldo Cruz, Avenida Brasil 4365, 21045-900, Rio de Janeiro, RJ, Brazil \\ ${ }^{2}$ Instituto Militar de Engenharia, Praça General Tibúrcio 80, 22290-270, Rio de Janeiro, RJ, Brazil \\ ah.schechtman@fiocruz.br, ${ }^{\mathrm{b}} \mathrm{mpelajo@ioc.fiocruz.br,}{ }^{\mathrm{c}} \mathrm{marceloprado@ime.eb.br,}$ \\ dcamuller@ioc.fiocruz.br, ’gurgeldeandrade@yahoo.com.br, 'henrique.lenzi@gmail.com
}

Keywords: Hidroxyapatite, Bone Marrow, Hematopoiesis, In Vivo, Rat.

\begin{abstract}
The extracellular matrix in skeletal tissue besides being responsible for its structure and function also provides key regulatory signals for cell proliferation and differentiation by interacting directly with cell receptors, controlling the diffusion of soluble growth factors. Immature bone marrow cells can migrate through capillaries to systemic circulation as well as return to bone marrow. This phenomenon is the biological basis for bone marrow transplant. The mechanisms that control the migration and the return of these immature bone marrow cells are still to be described in a generally accepted model.

Thirty days-old Wistar rats were employed as recipients for the implantation of ceramic blocks or demineralized femur diaphysis. The material was implanted subcutaneously at the dorsum. The rats were euthanized with $\mathrm{CO}_{2}$ at 15, 40 and 68 days after implantation. The whole area of implantation was harvested from skin deep up to the muscle. Liver, spleen, lungs, femurs and the vertebral spine, ribs and sternum were also harvested. The harvested material was fixed in Millonig formalin and decalcified with EDTA, if applicable. The material was then progressively dehydrated in alcohol, immersed in xylol, impregnated and embedded in paraffin. The paraffin blocks were then sliced in $5 \mu \mathrm{m}$ sections with a microtome, stained in Haematoxilin - Eosin, Lennert's Giemsa and Gomori's reticulin for histological analysis under brightfield optical microscopy.

All implants produced an intense fibroblastic reaction, angiogenesis and subsequent modulation into a loose stroma. However, hematopoesis was not observed. Implantation of endogenous haematopoetic cells in an inductive stroma, which might propitiate hematopoesis is a model scarcely studied. Experiments such as the one described here might be good models for understanding the sequence of events leading to formation of the stroma and implantation and differentiation of haematopoetic precursors.
\end{abstract}

\section{Introduction}

The extracellular matrix in skeletal tissue besides being responsible for its structure and function also provides key regulatory signals for cell proliferation and differentiation by interacting directly with cell receptors, controlling the diffusion of soluble growth factors [1].

Different approaches have been implemented to try and generate bone and bone marrow, some based on implanting pieces of bone, others a composite of collagen and Hidroxyapatite [1,2,3]. Generally speaking, the resulting effect of these implantations is the formation of bone and, sometimes, ectopic bone marrow. The most prominent features are angiogenesis and a loose stroma, propicious to hematopoesis.

Immature hematopoietic cells can migrate through capillaries to systemic circulation as well as return to bone marrow. This phenomenon is the biological basis for bone marrow transplant [4]. However, the mechanisms that control the migration and the return of these immature bone marrow cells are yet to be described in a generally accepted model. 
Three different aspects should be considered during this „homing“: the implanted scaffold itself, a hematopoietic progenitor release molecule and an attractor molecule.

\section{Materials and Methods}

The scaffolds used in this study are a patented product [5] that consists of an interconnected porous ceramic composed of hydroxyapatite and beta-TCP. The scaffolds were produced by sintering polymeric spoges coated with apatite at $1200^{\circ} \mathrm{C}$. The coatings were produced by hydrothermal precipitation of monetite on polymeric sponges and further conversion to hydroxyapatite. The resultant ceramic block scaffold, with dimensions of $2 \times 2 \times 1 \mathrm{~mm}$, exhibited a highly porous and interconnected structure, with an average pore size of $200 \mu \mathrm{m}$ (Fig. 1).

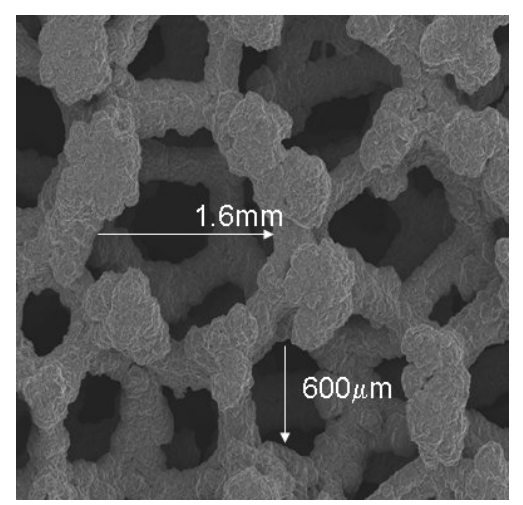

Figure 1 - Scanning electron microscopy image of the porous scaffold.

Whole rat femur dyaphisis were washed with distilled water, immersed into alcohol and ether for 30 and 60 minutes, respectively, and dryed at $37{ }^{\circ} \mathrm{C}$ for 10 hours. These bone segments were then demineralized in $0.5 \mathrm{~N}$ chloridric acid, again washed for 2 hours in distilled water, dehydrated in alcohol for 1 hour and ether for 30 minutes and finally dried at $37^{\circ} \mathrm{C}$.

Before implantation all material was submitted to ultraviolet light for 2 hours.

Thirty days-old Wistar rats were employed as recipients for the implantation of the ceramic blocks and the demineralized diaphysis. The material was implanted subcutaneously at the dorsum.

The rats were euthanized with $\mathrm{CO}_{2}$ at 15, 40 and 68 days after implantation. The whole area of implantation was harvested from skin deep up to the muscle. Liver, spleen, lungs, femurs and the vertebral spine, ribs and sternum were also harvested.

The harvested material was fixed in Millonig formalin and decalcified with EDTA, if applicable. The material was then progressively dehydrated in alcohol, immersed in xylol, impregnated and embedded in paraffin. The paraffin blocks were then sliced in $5 \mu \mathrm{m}$ sections with a microtome, stained in Haematoxilin - Eosin (HE), Lennert's Giemsa and Gomori's reticulin for histological analysis under brightfield optical microscopy.

\section{Results}

Representative micrographs of the tissue reaction to the implants are presented below. These micrographs of the HE stained tissue were taken at a magnification of 20x. Figures 2 and 3 present micrographs of the tissue reaction for the ceramic blocks at 15 and 68 days after implantation, respectively. Fig. 4 presents a micrograph of the tissue reaction for the diaphysis at 68 days after implantation.

Where ceramic implants were present, many giant cells, usually with more than 10 nuclei, were observed. At the interface of the giant cells with the implanted material, citoplasmatic heterogeneity was observed, indicating the resorption of the implanted material by these cells. 
It can also be observed the phenomenon of angiogenesis, with the presence of small and mediumcaliber vessels. This phenomenon appears to increase with time, as there were more vessels present at 68 than at 15 days. Scattered hemorrhagic foci were also detected, specially at 15 days.

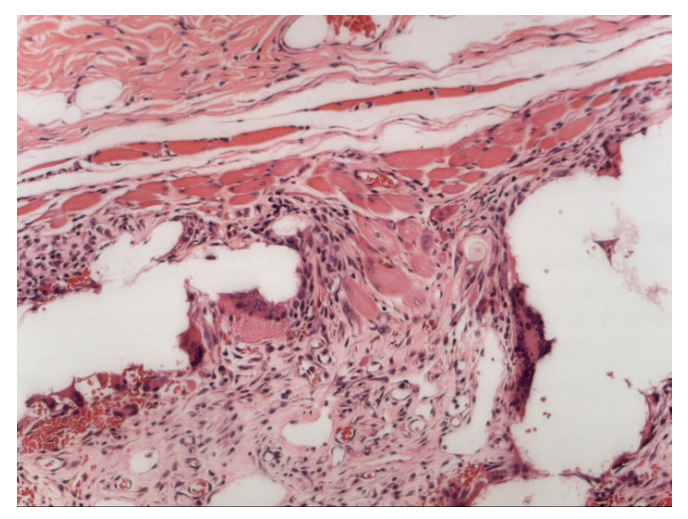

Figure 2 - Tissue reaction for the porous scaffold at 15 days after implantation. Magnification 20x, HE stained.

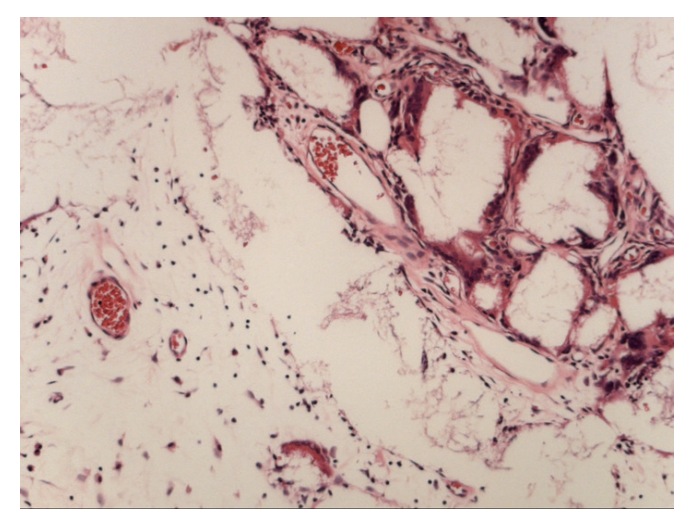

Figure 3 - Tissue reaction for the porous scaffold at 68 days after implantation. Magnification 20x, HE stained.

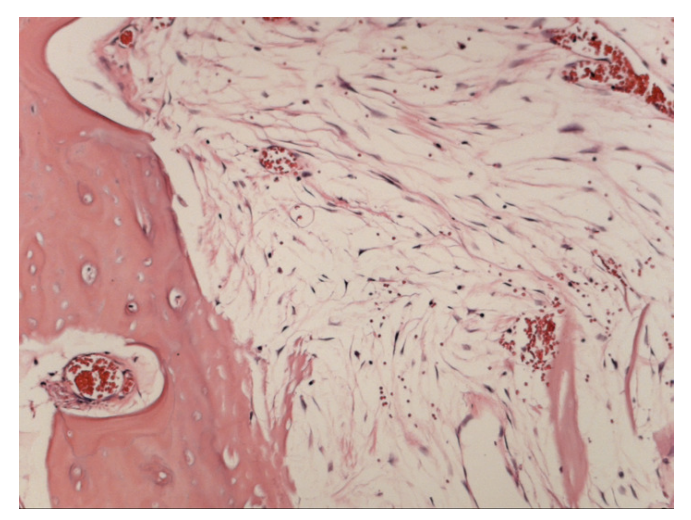

Figure 4 - Tissue reaction for the diaphysis at 68 days after implantation. Magnification 20x, HE stained.

Both ceramic and bone matrix implants initially induced proliferation of connective tissue that surrounded all the implant and even invaded the internal spaces. Subsequently, the whole tissue underwent internal epithelization and organization, where it was observed a modulation of the 
mesenchyma to a loose stroma, rich in vessels, with concomitant presence of osteoblasts and osteoclasts, with an appearance similar to an aplastic bone marrow. However, it is also observed, especially in Fig. 3, that no hematopoesis was present.

\section{Discussion and Conclusions}

Implantation of endogenous haematopoetic cells in an inductive stroma, which might propitiate hematopoesis is a model scarcely studied. Experiments such as the one described here might be good models for understanding the sequence of events leading to formation of the stroma and implantation and differentiation of haematopoetic precursors.

All implants initially induced intense proliferation of connective tissue, mainly from adjacent muscles, surrounding all the implant and even invading the internal spaces. Together with this fibroblast proliferation, an angiogenic process originated at vessels from the adjacent muscular tissue towards the newly-formed mesenchyma, was observed. Subsequently, the whole tissue underwent internal epithelization and organization, where it was observed a modulation of the mesenchyma to a loose stroma, rich in vessels, with concomitant presence of osteoblasts and osteoclasts, providing an appearance similar to an aplastic bone marrow. However, all the implants tested here failed to present hematopoiesis.

Moreover, implantation of the ceramic block strongly induced the presence and activity of giant cells, thus being an interesting model to study this cell biology.

An interesting event was the frequent occurrence of hemorrhagic foci surrounding the ceramic implant, especially at 15 days after implantation, which might be explained either by trauma following the surgical intervention for implantation, but also by late spontaneous capillary rupture. There were no histopathological changes in the organs harvested.

As far as these experimental systems failed to induce extramedullary hematopoiesis alone, further studies, employing progenitor cell migration factors and retention extracellular molecules, are being undertaken at our laboratories.

\section{References}

1. J.I. Dawson, D.A. Wahl, et al. Biomaterials 29 (2008), 3105-3116.

2. N.M. Ideriha, I. Vugman, and R.P. Falcão Blut 48 (1984), 277-284.

3. A.H. Reddi and C.B. Huggins Proc. Nat. Acad. Sciences 72 (1975), 2212-2216.

4. E.D. Thomas JAMA 235 (1976), 611-612.

5. M.H.P. Silva, S.R.A. Santos, and A.M. Rossi, Process for Porous Interconnected Substrate Coating, Synthesis Intermediate and Obtained Porous Product (2007). 